\title{
Localización del Septo del Seno Maxilar a través de Abordaje Endoscópico. Reporte de Caso y Revisión de la Literatura
}

\author{
Location of Maxillary Sinus Septum through an Endoscopic Approach. \\ Report of a Case and Review of the Literature
}

"Víctor Javier Beltrán Varas; "Ramón Fuentes Fernández; "*Wilfried Engelke; ${ }^{* * * *}$ Francisco Marchesani Carrasco \& *Marco Flores Velásquez

\begin{abstract}
BELTRÁN, V. V. J.; FUENTES, F. R.; ENGELKE, W.; MARCHESANI, C. F \& FLORES, V. M. Localización del septo del seno maxilar a través de abordaje endoscópico. Reporte de caso y revisión de la literatura. Int. J. Morphol., 29(2):636-643, 2011.

RESUMEN: El abordaje quirúrgico del seno maxilar para maniobras como el levantamiento de la membrana sinusal y la colocación de injerto óseo con o sin implantes simultáneo en la zona subantral, es un procedimiento cada vez más habitual en implantología oral. Una de las mayores complicaciones en este tipo de procedimientos es el abordaje de la pared lateral del seno maxilar con presencia de septos intra-sinusales no diagnosticados, pudiendo producir perforación de la membrana durante el fresado óseo. La presencia de septos en el seno maxilar es muy variable, pudiendo presentarse única o múltiple y en diferentes localizaciones anatómicas en relación a la zona de las raíces de las piezas maxilares antrales en pacientes dentados o desdentados, desde la zona canina hasta el segundo molar. Diversos autores, han descrito diferentes valores de la prevalencia en relación a la presencia de septos intra-sinusales en base a imagenología tridimensional o in vivo en estudios cadavéricos. El objetivo del presente estudio, es realizar una revisión exhaustiva de la literatura desde 1997 hasta noviembre del 2010 en relación a la prevalencia de septos en el seno maxilar y su localización según método de visualización, y el reporte de un caso con una nueva técnica de abordaje quirúrgico que permite una visualización intra y extrasinusal para la localización del septo del seno maxilar in vivo.
\end{abstract}

PALABRAS CLAVE: Seno maxilar; Septo del seno maxilar; Anatomía.

\section{INTRODUCCIÓN}

La elevación de seno maxilar es un procedimiento de rutina en implantología oral, considerándose una técnica quirúrgica predecible y segura, con o sin la colocación inmediata de implantes endoóseos dependiendo esto de la altura del reborde alveolar remanente, lo que requiere una planificación prequirúrgica tridimensional (Neugebauer $e t$ $a l ., 2010)$. Una de las mayores complicaciones que se presenta en este tipo de abordaje, es la presencia de uno o más septos intrasinusales, siendo de prevalencia y localización variable y de difícil diagnóstico imagenológico convencional (Kreinnmair et al., 1997; Kim et al., 2006; Shibli et al., 2007; Ella et al., 2008; Koymer et al., 2009; Gosau et al., 2009; Naitoh et al., 2009; Van Zyl \& Van Heerden, 2009; Lee et al., 2010; Neugebauer et al., 2010; Rosano et al., 2010).

Los septos intrasinusales pueden ser definidos como una formación de hueso cortical dentro del seno maxilar, con forma de arco y que se pueden originar de la pared inferior o lateral del seno maxilar, pudiendo a su vez dependiendo de su tamaño, dividir el seno maxilar en dos o más cavidades (Underwood, 1910) .

La presencia de septos del seno maxilar se puede explicar como un fenómeno resultante del desarrollo de los maxilares o producto de una irregular neumatización por pérdida prematura de piezas dentarias (Krennmair et al., 1999). No considerar la posible presencia de esta variación anatómica previo a la elevación de seno maxilar, implica necesariamente un riesgo elevado de la localización de la membrana sinusal, pudiendo producir perforaciones accidentales de ésta y hemorragia por ruptura de arteriolas en la zona anatómica (Krennmair et al., 1997; Neugebauer et al., 2010).

\footnotetext{
* Departamento de Odontología Integral, Facultad de Medicina, Universidad de La Frontera, Temuco, Chile.

** Department of Oral Surgery, School of Dentistry, Georg-August-University Gottingen, Germany.

**** Unidad de Implantología, Facultad de Odontología, Universidad de Concepción, Concepción, Chile.
} 
La imagenología tridimensional ha permitido una localización exacta de éstas variaciones, permitiendo una planificación quirúrgica óptima. Sin embargo, ello no implica que el abordaje quirúrgico sea sencillo, ya que la visualización directa del cirujano en el sitio anatómico es compleja y poco accesible. En este sentido, la introducción de la endoscopía en la implantología a través de los últimos años, ha permitido la visualización de cavidades óseas cerradas, estructuras neuro-vasculares y posiciones ectópicas de piezas dentarias (Engelke \& Galle, 2008).

Engelke et al. (2003) describen un procedimiento mínimamente invasivo para el abordaje del espacio subantral, con una osteotomía mínima de la pared ósea lateral para una tunelización laterobasal a través de un endoscopio con soporte rígido y decoladores microquirúrgicos. Opcionalmente podemos realizar un acceso simultáneo a través de la pared anterior del seno maxilar inmediatamente por debajo del foramen infraorbitario, para tener una visión al interior del seno maxilar permitiendo monitorizar la membrana sinusal en su porción interna y externa.

Presentamos el reporte de un caso con una técnica de visualización in vivo, tridimensional y mínimamente invasiva a través del endoscopio. Se pondrá énfasis en nombrar las zonas quirúrgicas en relación a la Terminología Anatómica.

Se realizó una búsqueda de la literatura utilizando PubMed a través de diferentes palabras claves, en estudios descriptivos como series de casos o de corte transversal.

\section{MATERIAL Y MÉTODO}

Se describe el reporte de un caso de localización de un septo intra-sinusal en el seno maxilar izquierdo, para un procedimiento de elevación de seno y posterior colocación de implantes endoóseos, utilizando la técnica SALSA (Engelke et al.), que consiste en un abordaje a través de un endoscopio (Fig.1.A y 1.B), con tunelización latero-basal para una visualización externa de la membrana y del espacio subantral, y paralelamente un abordaje intra-sinusal accesorio a través de trocar micro-quirúrgico por debajo del foramen infraorbitario (Fig.2.A y 2.B), para un monitoreo interno de la membrana sinusal, durante la remoción del septo por fresado óseo.

Se realizó una búsqueda de la literatura disponible utilizando PubMed, a través de diferentes palabras claves: seno maxilar, sinus maxillary anatomy, septo del seno maxilar, maxillary sinus septa, sinus lift. La búsqueda fue conducida el 11 de Noviembre del 2010. De la selección final de artículos sólo fueron considerados estudios descriptivos como series de casos o de corte transversal desde el año 1997 hasta el 2010.

Se seleccionaron aquellos artículos que describen la metodología utilizada en la visualización de los septos del seno maxilar (Tabla I), y en relación a la prevalencia total de septos según el total de pacientes o cadáveres utilizados y del total de senos de la muestra estudiada.
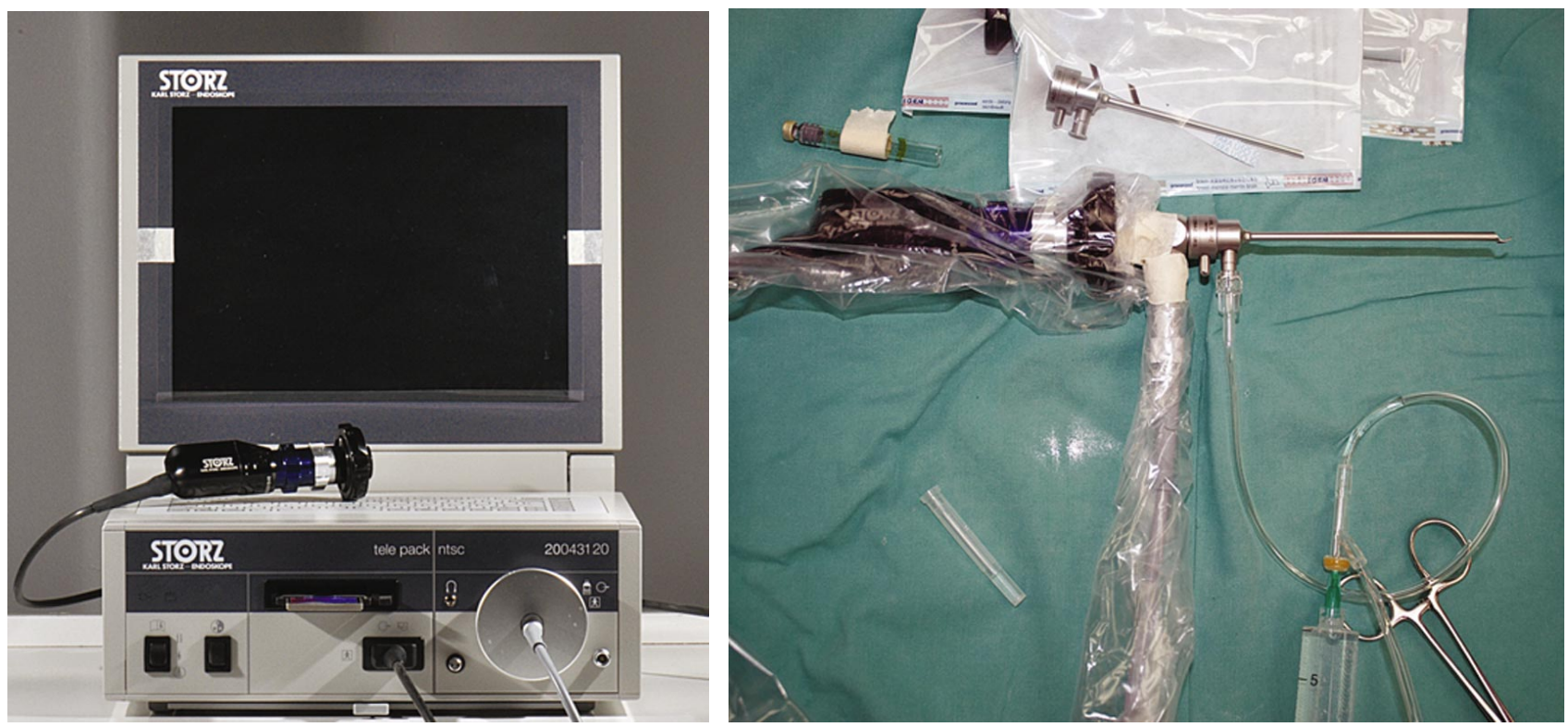

Fig. 1.A. Monitor; Fig.1.B. endoscopio acoplado listos para usarse en cirugía oral. 

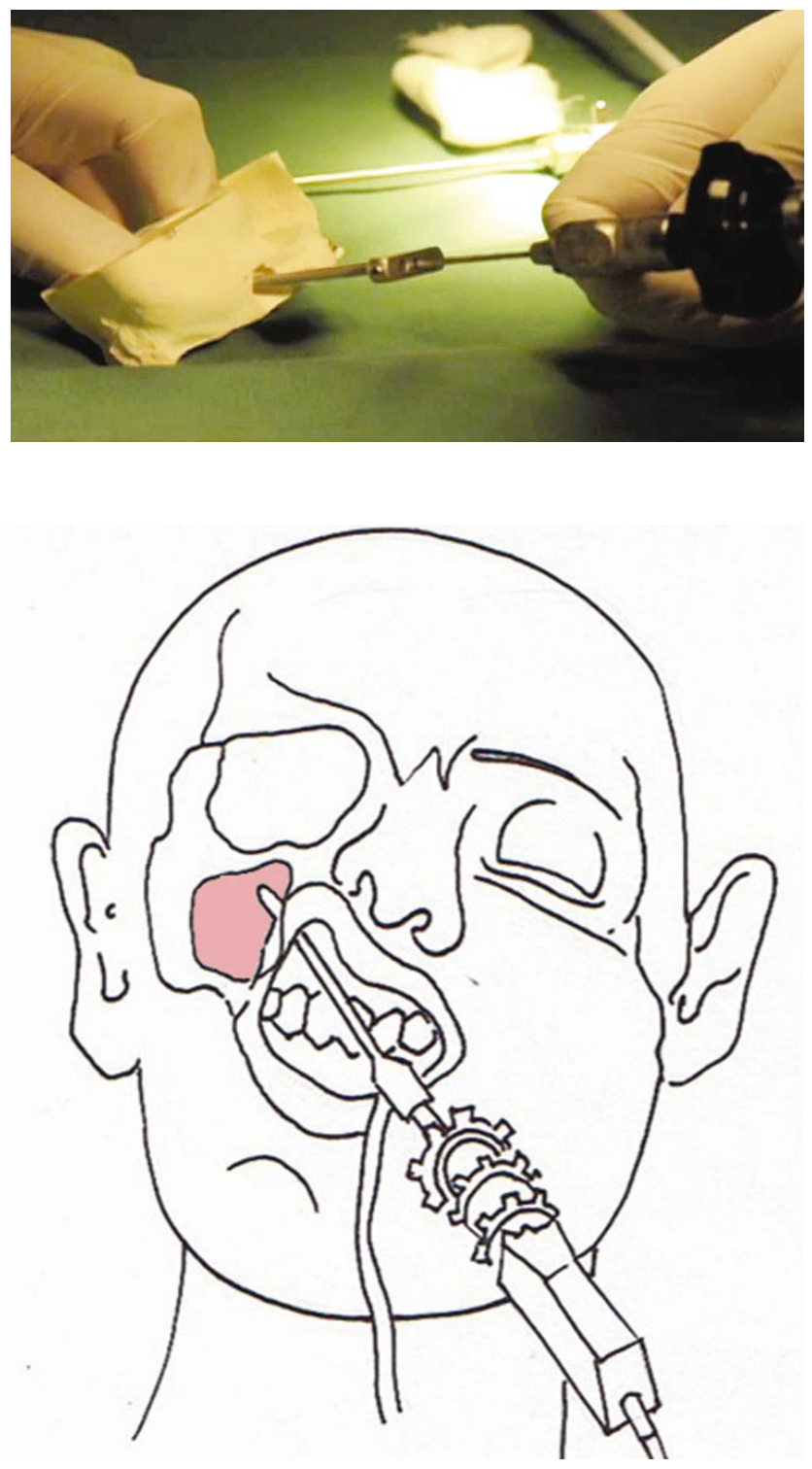

Fig. 2.A. Abordaje intra-sinusal endoscópico, para tener una visualización del interior de la membrana sinusal. La imagen representa la inserción de la óptica endoscópica en el interior del seno maxilar. Fig.2.B.Este abordaje se realiza a través de un trocar microquirúrgico en la zona inmediatamente inferior a la fosa canina, intra-oralmente perforando la mucosa del vestíbulo y la membrana sinusal en un diámetro de $2,7 \mathrm{Mm}$.

Un total de 13 artículos fueron finalmente seleccionados y analizados del total de la búsqueda. Fueron excluidos aquellos artículos que a pesar de describir los parámetros en discusión, presentaban simultáneamente un análisis de dos técnicas de visualización al mismo tiempo, por no presentar o describir claramente el análisis de confiabilidad inter-observador que utilizaron los examinadores.
Tabla I. Método utilizado para la visualización de los septos del seno maxilar.

\begin{tabular}{lll}
\hline Autor & Año & Método de visualización \\
Krennmair et al. & 1997 & Tomografía Computarizada \\
Kim et al. & 2006 & Tomografía Computarizada \\
Shibli et al. & 2007 & Radiografía Panorámica. \\
Ella et al. & 2008 & Tomografía Computarizada \\
Koymer et al. & 2009 & Tomografía Computarizada \\
Gosau et al. & 2009 & In situ (cadavérico) \\
Naitoh et al. & 2009 & Tomografía Computarizada \\
Van Zyl et al. & 2009 & Tomografía Computarizada \\
Lee et al. & 2010 & Tomografía Computarizada \\
Neugebauer & 2010 & Tomografía Computarizada \\
Naitoh et al. & 2010 & Tomografía Computarizada \\
Rosano et al. & 2010 & In situ (cadavérico) \\
Beltrán et al. & 2011 & T. Computarizada/Endoscópico
\end{tabular}

\section{REPORTE DE CASO}

En una paciente de sexo femenino de 41 años, sistémicamente sana, se le solicitó una tomografía axial computarizada en el maxilar, para la planificación pre-quirúrgica de una elevación de seno maxilar izquierdo para la colocación diferida de implantes dentales endoóseos en dicha zona desdentada. La paciente ingresó en el Policlínico de Implantología del Dpto. de Odontología Integral de la Universidad de La Frontera, y fue intervenida en el pabellón quirúrgico de la Clínica Odontológica Docente Asistencial, implementada para cirugía mínimamente invasiva y bajo consentimiento informado.

A través de la tomografía axial computarizada, se detectó la presencia de un septo óseo intra-sinusal en relación al seno maxilar izquierdo (Fig. 3.A y 3.B). Se decidió realizar un abordaje para elevación del seno maxilar izquierdo a través de técnica SALSA o abordaje endoscópico subantral para la aumentación del piso del seno maxilar (Engelke, 2002) y simultáneamente un abordaje intra-sinusal endoscópico (Fig. 4.A y 4.B.), para mantener un monitoreo permanente de la indemnidad de la membrana sinusal y la realización de una osteotomía controlada en la pared anterior del seno maxilar, hasta lograr la visualización completa del septo del seno maxilar izquierdo para su remoción por fresado.

En este reporte, el septo del seno maxilar se encuentra localizado en la zona media de la extensión mesio-distal del seno maxilar izquierdo (zona del primer molar), con una 
altura aproximada de $7 \mathrm{Mm}$. (Fig. 3.A ), ampliamente extendido en sentido latero-medial (Fig. 3.B). Se realizan exodoncias múltiples y legrado quirúrgico de lesión periapical en relación al primer premolar izquierdo. Posteriormente, se realiza abordaje intra-sinusal a través de trocar metálico, para la inserción de óptica endoscópica de 2,7 $\mathrm{Mm}$. de diametro, $30^{\circ}$ de angulación, con fibra óptica sin irrigación, y las imágenes fueron registradas por un procesador de imagen digital y monitor (Karl Storz, Tuttlingen, Alemania), (Figs. 1.A y 1.B).

Posteriormente se realiza un colgajo muco-perióstico, para tener acceso a la pared lateral del seno maxilar. En este momento, se efectúa una osteotomía controlada en la pared lateral del seno maxilar (Fig. 5.A) para tener acceso a la porción externa de la membrana sinusal (Fig. 5.B). Con apo-
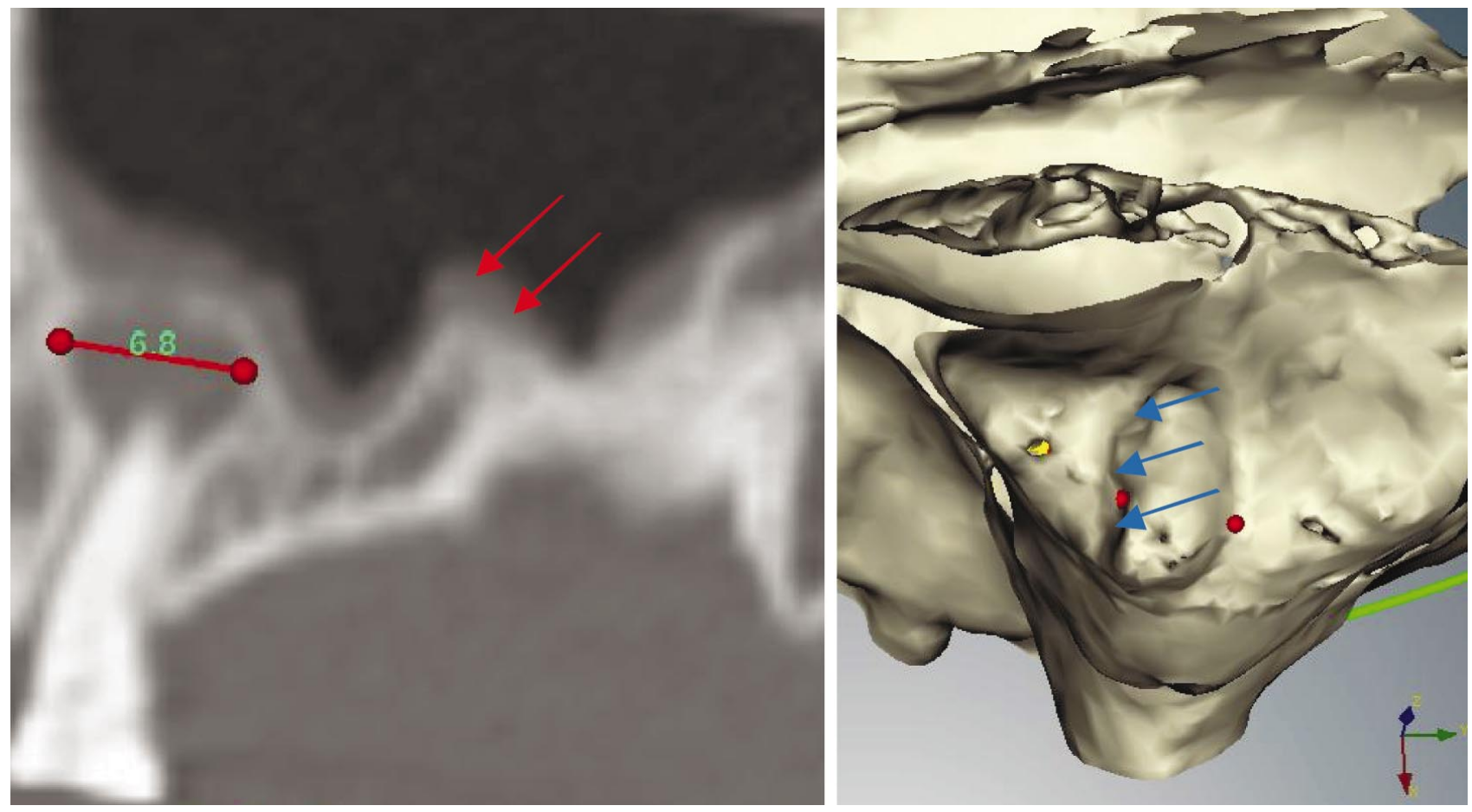

Fig. 3A. Visión panorámica del seno maxilar izquierdo. Se aprecia una amplia neumatización y la presencia de un quiste periapical en relación al primer premolar izquierdo. Las flechas rojas indican la presencia del septo del seno maxilar izquierdo con una altura aproximada de $7 \mathrm{Mm}$. en posición media (entre el primer y segundo molar). Fig. 3.B. Visión 3D superior del seno maxilar izquierdo (DentaScan), las flechas azules indican la extensión del septo intra-sinusal en sentido latero-medial.
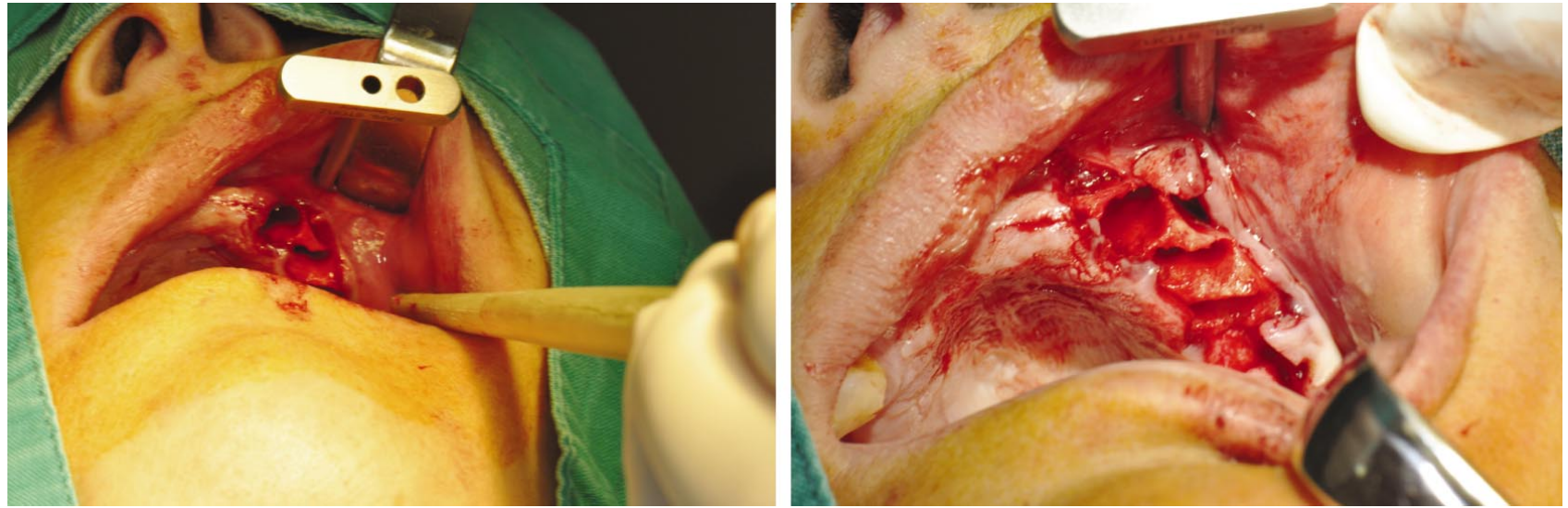

Fig. 4.A. Posicionamiento de Trocar para óptica de 2,7 Mm. (Karl Storz), en la pared anterior del seno maxilar izquierdo para permitir una visualización intra-sinusal. Fig.4.B.Es necesario proteger el paquete vasculo-nervioso infraobitario a través del dedo índice del cirujano, para evitar una lesión a éste. (Ver visualización endoscópica en Fig. M.) 

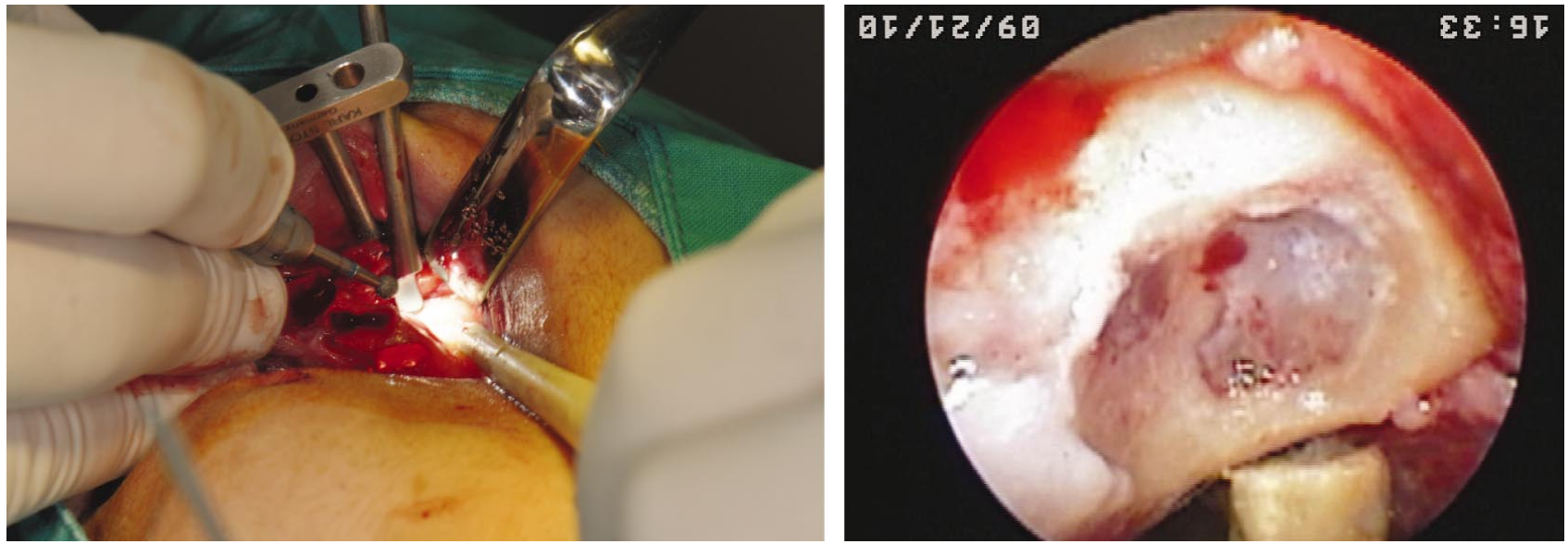

Fig. 5.A. Se realizó técnica SALSA para elevación de seno maxilar izquierdo descrita por Engelke (2002). Una vez concluida la osteotomía controlada a través de la pared lateral del seno maxilar, se realiza una tunelización en dirección latero-basal para permitir la inserción de la óptica endoscópica en el espacio subantral. Nótese según la flecha blanca, el soporte rígido que contiene la óptica endoscópica. Fig. 5.B. Visualización endoscópica macroscópica (óptica Karl Storz de 2,7 Mm. con soporte rígido y fibra óptica), la flecha negra muestra la clara visualización de la membrana sinusal en su porción externa, una vez concluida
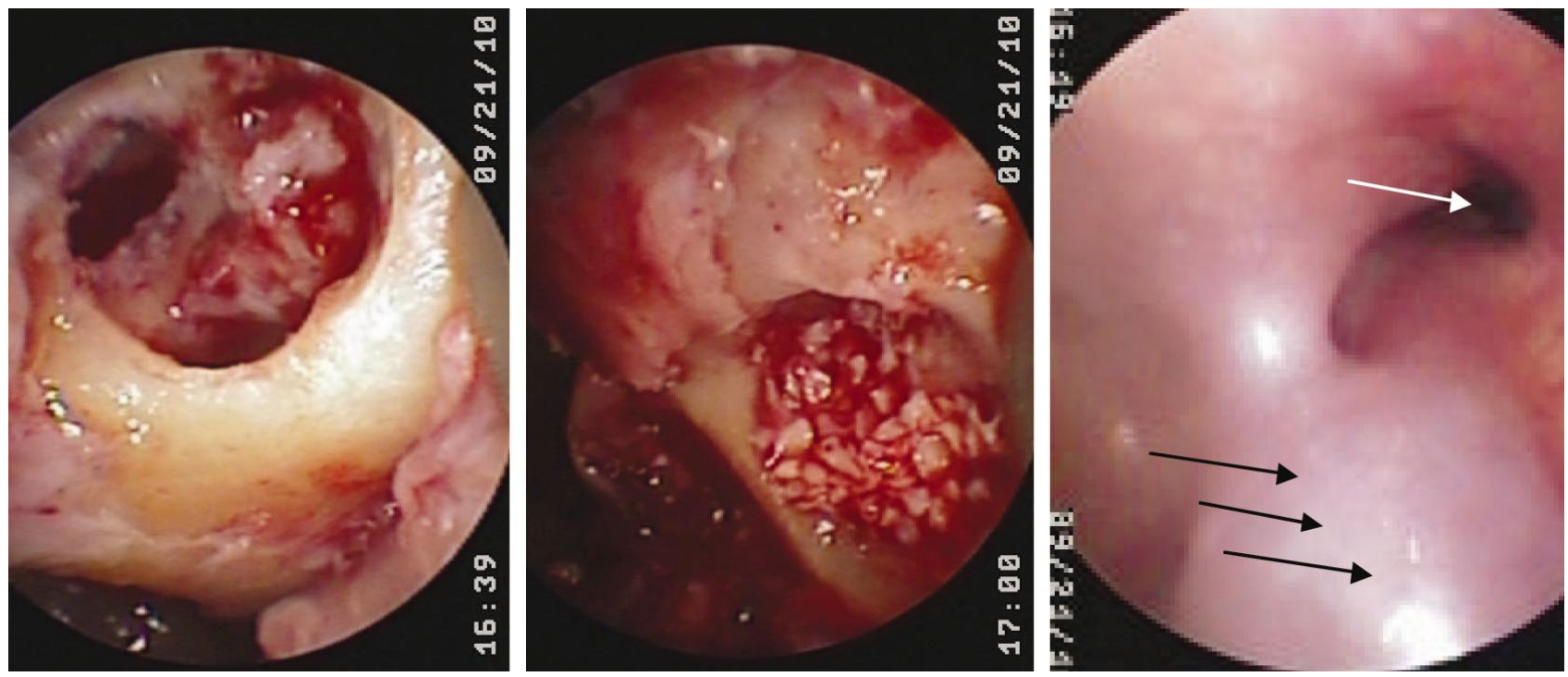

Fig. 6.A. Visualización endoscópica macroscópica del septo en el seno maxilar izquierdo. Se ve la extensión del septo intra-sinusal en sentido apico-coronal (ver flechas blancas), una vez que se ha despegado la membrana sinusal para el acceso al espacio subantral. Fig 6.B. Eliminación del septo intra-sinusal por fresado y colocación de injerto óseo particulado en el espacio subantral.

Fig. 6.C. Visualización endoscópica macroscópica en el espacio intra-sinusal una vez concluido el levantamiento del seno maxilar izquierdo. Se aprecia el ostium del seno maxilar (Flecha blanca). Se observa además, el levantamiento de la mucosa sinusal, sin perforación de ésta, por la interposición de injerto óseo particulado en el espacio subantral tras la eliminación del tabique. (Flechas negras).

yo endoscópico, a través de un soporte rígido que contiene la óptica de $2,7 \mathrm{Mm}$. de diámetro, $30^{\circ}$ de angulación y permite realizar irrigación continua a través del mismo. El procesador de imágenes permite a través de un monitor (Fig. A) visualizar la porción externa de la membrana sinusal para su despegamiento y acceso al espacio subantral, a la vez que se controla simultáneamente la indemnidad de la porción interna con el soporte endoscópico posicionado a tra- vés del trocar en la pared anterior del seno maxilar por debajo del penacho infraorbitario, es decir, logramos una visualización externa e interna de la membrana sinusal al mismo tiempo (Fig. 5.B y 6.C).

Una vez concluido el despegamiento de la membrana sinusal, se logra la visualización endoscópica del septo del seno maxilar a través de la óptica endoscópica en el es- 
Tabla II. Resumen de la prevalencia de septos del seno maxilar según el total de pacientes o cadáveres (Prevalencia \% del total de pacientes o cadáveres), y del total de senos maxilares de la muestra (Prevalencia \% del total de senos maxilares) y localización anatómica en relación a la zona de las piezas antrales (Localización mas prevalente).

\begin{tabular}{|c|c|c|c|c|c|}
\hline Autor & Año & $\begin{array}{l}\text { Número de } \\
\text { pacientes o } \\
\text { cadáveres }\end{array}$ & $\begin{array}{l}\text { Prevalencia \% del } \\
\text { total de pacientes o } \\
\text { cadáveres }\end{array}$ & $\begin{array}{l}\text { Prevalencia \% } \\
\text { del total de } \\
\text { senos maxilares }\end{array}$ & Localización más prevalente \\
\hline Krennmair et al. & 1997 & 165 & - & 18,8 & Región de primer y segundo \\
\hline Kim et al. & 2006 & 100 & 26,5 & - & Región primer y segundo molar \\
\hline Shibli et al. & 2007 & 1024 & 21,58 & - & - \\
\hline Ella et al. & 2008 & 75 & 39 & - & - \\
\hline Koymer et al. & 2009 & 205 & 35,37 & - & Región del primer y segundo \\
\hline Gosau et al. & 2009 & 65 & - & 27 & Región primer molar. \\
\hline Naitoh et al. & 2009 & 42 & - & 30 & - \\
\hline Van Zyl et al. & 2009 & 200 & 69 & - & - \\
\hline Lee et al. & 2010 & 204 & 27 & 24,6 & Región primer y segundo molar \\
\hline Neugebauer et al. & 2010 & 1029 & 47 & 33,2 & Región primer molar \\
\hline Naitoh et al. & 2010 & 44 & - & 41,7 & - \\
\hline Rosano et al. & 2010 & 60 & - & 33,3 & Región primer y segundo molar \\
\hline Beltrán et al. & 2011 & 1 & - & - & Región primer y segundo molar \\
\hline
\end{tabular}

pacio subantral (Fig. 6.A). En esta visualización endoscópica apreciamos la extensión del tabique en sentido latero-medial y ápico-coronal, lo que nos permite realizar la eliminación del septo con un fresado óseo controlado a través del monitor en tiempo real, reduciendo los riesgos de perforación de la membrana sinusal o ruptura de arteriolas en relación al territorio anatómico. Posteriormente, se procede al relleno del espacio subantral con injerto óseo particulado alogénico (Fig. 6.B), y control endoscópico simultáneo de la porción interna de la membrana sinusal y su relación con el ostium del seno maxilar izquierdo (Fig. 6.C).

Finalizada esta maniobra, se procede a la remoción del trocar y se sutura la pequeña perforación resultante, posteriormente se sutura el resto del colgajo muco-perióstico.

\section{DISCUSIÓN}

La prevalencia del septo en el seno maxilar, puede ser calculada basándose en número total de pacientes o cadáveres que presentan septos en el seno maxilar o en el número total de senos maxilares que presentan septos.

En estudios basados en el número de pacientes o cadáveres que presentan septos en el seno maxilar, la prevalencia varía entre 21,58 y $69 \%$. En estudios basados en el número de senos maxilares que presentan, la prevalencia varía entre 24,6 y 41,7\% (Tabla II). La metodología de visualización de elección es la tomografía computarizada (Tabla I).
Según Naitoh et al. (2010) la prevalencia de septos del total de senos maxilares incluidos en su estudio es superior al $40 \%$, no describiendo la localización mas prevalente. Sin embargo otros autores como Krennmair et al. (1997), reportan una prevalencia mucho más baja, no superior al $20 \%$, reportando como localización más frecuente la región de primer y segundo premolar, todos estos autores utilizaron la tomografía computarizada como herramienta diagnóstica, lo que da una certeza muy aproximada de los hallazgos reportados (Tabla II).

Es interesante recalcar la investigación llevada a cabo por Neugebauer et al. (2010), por la cantidad de pacientes incluidos (1029 pacientes) y determinando la localización más frecuente a nivel de una zona más posterior del seno maxilar, como lo es el primer molar, diferente a lo encontrado en otras descripciones anteriores. Aspectos importantes que podrían interferir en estos resultados, como es la condición étnica y el grado de desdentamiento prematuro de la población en estudio, generalmente no son reportados en la mayoría de los estudios. Estudios como los de Rosano et al. (2010) y Gosau et al. (2009) se realizaron en cadáveres, obteniendo resultados similares en cuanto a la prevalencia de septos según el total de senos maxilares pero diferentes en cuanto a la localización, los primeros describiendo una localización más posterior a nivel del primer y segundo molar y los segundos autores una un poco más anterior a nivel del primer molar, lo que coincide con el caso presentado (Tabla II).

La detección de septos óseos en el seno maxilar es posible a través de tomografía computarizada de manera 
confiable. Diversos autores reportan que la radiografía panorámica induce a error en la detección de septos, sobre todo a verdaderos negativos (Kreinnmair et al., 1999; Kasabah et al., 2002; González-Santana et al., 2007).

La incorporación de herramientas de planificación quirúrgica en base a la imagenología tridimensional, ha tenido un desarrollo explosivo en los últimos 5 años. Por consiguiente, la elevación de seno maxilar ha ido evolucionando de una técnica traumática a una técnica mínimamente invasiva con mejores consecuencias post-quirúrgicas para el paciente.

En este sentido la visualización endoscópica en tiempo real, presentada en este caso, de estructuras de difícil acceso al cirujano con las técnicas convencionales, nos permiten la visualización de estructuras anatómicas críticas al campo quirúrgico y de variaciones anatómicas como es el caso de la presencia de septos intra-sinusales. Al utilizar esta herramienta de visualización en tiempo real, complemen- tando la técnica de SALSA con una visión endoscópica interna del seno maxilar para una mejor monitorización de la membrana sinusal, permite una visualización y abordaje quirúrgico minimamente invasivo que confirma de manera exacta la información entregada por la imagenología tridimensional.

Previamente se realizó una revisión sistemática de la prevalencia y localización de septos en el seno maxilar (Maestre-Ferrín et al., 2010). Sin embargo, esta revisión fue realizada hasta Julio del 2008, no considerando la prevalencia de septos intrasinusales en estudios cadavéricos, y no excluyendo artículos que usaban dos métodos de visualización en los mismos pacientes, sin un método de confiablidad inter-observador descrito. La revisión que nosotros presentamos, contempla un desarrollo mayor de investigaciones en esta línea, dos años posteriores a esta revisión, incluyendo análisis cadavéricos in situ o a través de imagenología tridimensional.

BELTRÁN, V. V. J.; FUENTES, F. R.; ENGELKE, W.; MARCHESANI, C. F \& FLORES, V. M. Location of maxillary sinus septum through an endoscopic approach. Report of a case and review of the literature. Int. J. Morphol., 29(2):636-643, 2011.

SUMMARY: Surgery of the maxillary sinus in procedures as elevation of the sinus membrane and bone graft, with or without simultaneous implants in the subantral area, is becoming a more standard procedure in oral implants each time. One of the most common complications in this type of procedure is the approach of the sinus lateral wall, with presence of undiagnosed intra-sinus septa with perforation of the membrane during sinus elevation. The presence of septa in the maxillary sinus is variable, presenting as single or multiple in different locations with relation to the rots in maxillary pieces in dentate or edentulous patients. Various authors have described different values in the prevalence of the intra sinus septum presence, based on three dimensional imaging studies, or the in vivo study of cadavers. The aim of this study is an exhaustive review of the literature since 1997 to November of 2010 in reference to the prevalence of maxillary sinus septa and their location according to observation methods, and the report of a case with innovative surgical approach technique, that allows intra and extra sinus visualization for in vivo maxillary sinus septum location.

KEY WORDS: Maxillary sinus; Maxillary sinus septum; Anatomy.

\section{REFERENCIAS BIBLIOGRÁFICAS}

Ella, B.; Noble, C.; Lauverjat, Y.; Sédarat, C.; Zwetyenga, N.; Siberchicot, F. \& Caix, P. Septa within the sinus: effect on elevation of the sinus floor. J. Oral Maxillofac. Surg., 46(6):464-7, 2008

Engelke, W.; Schwarzäller, W.; Behnsen, A. \& Jacobs, H.G. Subantroscopic Laterobasal Sinus Floor Augmentation (SALSA): An Up-to-5 Year Clinical Study. Int. J. Oral Maxillofac. Implants, 18(1):135-43, 2003.

Engelke, W. In situ Examination of Implant Sites with Support Immersion Endoscopy. Int. J. Oral Maxillofac. Implants, 17(5):703-6, 2002.
Engelke, W. \& Galle, C. Endoscopic Interventions in Oral Implantology - A Report of 5 Years Clinical Experience. Int. J. Odontostomat., 2(2):153-61, 2008.

González-Santana, H.; Peñarrocha-Diago, M.; GuarinosCarbó, J. \& Sorni-Bröker, M. A study of the septa in the maxillary sinuses and the subantral alveolar processes in 30 patients. J. Oral Implantol., 33(6): 3407, 2007.

Gosau, M.; Rink, D.; Driemel, O. \& Draenert, F.G. Maxillary Sinus Anatomy: A Cadaveric Study With Clinical Implications. Anat Rec., 292:352-354, 2009. 
Kasabah, S.; Slezák, R.; Simunek, A.; Krug, J. \& Lacaro, M.C. Evaluation of the accuracy of panoramic radiograph in the definition of maxillary sinus septa. Acta Medica, 45(4):173-5, 2002.

Kim, M.J.; Jung, UW.; Kim, CS.; Kim, K.D.; Choi, S.H.; Kim, C.K. \& Cho, K.S. Maxillary sinus septa: prevalence, height, location, and morphology. A reformatted computed tomography scan analysis. $J$. Perodontol., 77(5):903-908, 2006.

Koymen, R.; Gocmen-Mas, N.; Karacayli, U.; Ortakoglu, K.; Ozen, T. \& Yazici, A.C. Anatomic evaluation of maxillary sinus septa: surgery and radiology. Clin Anat., 22(5):563-570, 2009.

Krennmair, G.; Ulm, C. \& Lugmayr, H. Maxillary sinus septa: incidence, morphology and clinical implications. J. Craniomaxillofac. Surg., 25(5):261-5, 1997.

Krennmair, G.; Ulm, C.; Lugmayr, H. \& Solar, P. The incidence, location, and height of sinus septa in the edentulous and dentale maxilla. J. Oral Maxillofac. Surg., 57(6): 667-71, 1999.

Lee, W.; Lee, S. \& Kim, H. Analysis of location and prevalence of maxillary sinus septa. J. Periodontal Implant, 40:56-60, 2010.

Maestre-Ferrín, L.; Galán-Gil, S.; Rubio-Serrano, M.; Peñarrocha-Diago, M. \& Peñarrocha-Oltra D. Maxillary sinus septa: A systematic review. Med. Oral Patol. Oral Ci.r Bucal, 15 (2):383-6, 2010.

Naitoh, M.; Suenaga, Y.; Kondo, S.; Gotoh, K. \& Ariji, E. Assesment of maxillary sinus septa using cone-beam computed tomography: etiological consideration. Clin. Implant. Dent. Relat. Res., 1:52-58, 2009.

Naitoh, M.; Suenaga, Y.; Gotoh, K.; Ito, M.; Kondo, S. \& Ariji, E. Observation of maxillary sinus septa and bony bridges using dry skulls between Hellman' s dental age of IA and IIIC. Okajimas Folia Anat. Jpn., 87(2):41-7, 2010.

Neugebauer, J.; Ritter, L.; Mischkowsky, R.A.; Dreiseidler, T.; Scherer, P.; Katterle, M.; Rothamel, D. \& Zoller, J.E. Evaluation of maxillary sinus anatomy by cone beam CT prior to sinus floor elevation. Int. J. Oral Maxillofac. Implants., 25(2):258-65, 2010.

Rosano, G.; Taschieri, S.; Gaudy, J.F.; Lesmes, D. \& Del Fabbro, M. Maxillary sinus septa: a cadaveric study. $J$. Oral Maxillofac. Surg., 68(6):1360-1364, 2010.
Shibli, J. A.; Faveri, M.; Ferrari, D. S.; Melo, L.; García, R.V.; Avila, S.; Figueiredo, L. C. \& Feres, M. Prevalence of maxillary sinus septa in 1024 with edentulous upper jaws: a retrospective study. J. Oral Implantol., 33(5):2936, 2007.

Underwood, A. S. An inquiry into the anatomy and pathology of the maxillary sinus. J. Anat. Physiol., 44:354-69, 1910.

Van Zyl, A.W. \& Van Heerden, W.F. A retrospective analysis of maxillary sinus septa on reformatted computerized tomography scans. Clin. Oral Implants Res., 20(12):1398-401, 2009.

Dirección para correspondencia:

Dr. Víctor Beltrán Varas

Dpto. Odontología Integral

Facultad de Medicina

Universidad de La Frontera

Calle Claro Solar N ${ }^{\circ} 115$-Of. 420

Temuco

CHILE

Email: vbeltranvaras@gmail.com

Recibido : 06-01-2011

Aceptado: 26-02-2011 